\title{
DISTANCE BETWEEN NORMAL OPERATORS
}

\author{
V. S. SUNDER ${ }^{1}$
}

ABSTRACT. Lidskii and Wielandt have proved independently that if $A$ and $B$ are selfadjoint operators on an $n$-dimensional space $H$, with eigenvalues $\left\{\alpha_{k}\right\}_{k=1}^{n}$ and $\left\{\beta_{k}\right\}_{k=1}^{n}$ respectively (counting multiplicity), then,

$$
\|A-B\| \geqslant \min _{\sigma \in S_{n}}\left\|\operatorname{diag}\left(\alpha_{k}-\beta_{\sigma(k)}\right)\right\|
$$

for any unitarily invariant norm on $L(H)$. In this note an example is given to show that this result is no longer true if $A$ and $B$ are only required to be normal (even unitary). It is also shown that the above inequality holds in the operator norm, if $A$ is selfadjoint and $B$ is skew-self-adjoint.

Introduction. In [2], Weyl proved the following: if $A$ and $B$ are Hermitian operators on a finite-dimensional Hilbert space, and if $\operatorname{sp} A=\left\{\alpha_{k}\right\}_{k=1}^{n}$, $\operatorname{sp} B=\left\{\beta_{k}\right\}_{k=1}^{n}$ (counting multiplicity), then

$$
\|A-B\| \geqslant \min _{\sigma \in S_{n}}\left\|\operatorname{diag}\left(\alpha_{k}-\beta_{\sigma(k)}\right)\right\| .
$$

(Here, $\operatorname{sp} A$ denotes the spectrum of $A$, and $\operatorname{diag}\left(\gamma_{k}\right)$ denotes any normal operator with spectrum $\left\{\gamma_{k}\right\}_{k=1}^{n}$.) The norm used above is the operator norm. It was later shown by Lidskii [1] and Wielandt [3] that (*) is valid in any norm on $L(H)$ that is unitarily invariant. (Throughout this note, $H$ denotes a finite-dimensional Hilbert space.)

In this short note, we first give an example to show that the analogue of Lidskii's theorem for general normal operators is false. In the example given, $A$ is Hermitian and unitary, while $B$ is skew-Hermitian and unitary. We show, however (cf. Theorem 2) that Weyl's theorem remains valid if $A$ is Hermitian and $B$ is skew-Hermitian.

EXAMPLE 1. Let

$$
A=\left[\begin{array}{ll}
0 & 1 \\
1 & 0
\end{array}\right], \quad B=\left[\begin{array}{cc}
0 & -1 \\
1 & 0
\end{array}\right] .
$$

Then $A$ is Hermitian, $B$ is skew-Hermitian and $\operatorname{sp} A=\left\{\alpha_{1}, \alpha_{2}\right\}, \operatorname{sp} B=\left\{\beta_{1}, \beta_{2}\right\}$ where $\alpha_{1}=1, \alpha_{2}=-1, \beta_{1}=i, \beta_{2}=-i$. (Note that $A$ and $B$ are unitary.) If $\|\cdot\|_{p}$ is the Schatten $p$-norm, then it is easily checked that

$$
\|A-B\|_{p}=2, \quad\left\|\operatorname{diag}\left(\alpha_{k}-\beta_{\sigma(k)}\right)\right\|_{p}=2^{1 / 2+1 / p},
$$

for any $\sigma$ in $S_{2}$. Thus, if $1 \leqslant p<2$,

$$
\|A-B\|_{p}<\min _{\sigma \in S_{2}}\left\|\operatorname{diag}\left(\alpha_{k}-\beta_{\sigma(k)}\right)\right\|_{p} .
$$

Received by the editors May 11, 1981.

1980 Mathematics Subject Classification. Primary 15A60, 15A57, 65F35; Secondary 15A42, 47A55.

${ }^{1}$ The author is currently a Visiting Fellow at the Tata Institute of Fundamental Research. 
In the above example, the operator norm of $A-B$ is larger than $\min _{\sigma \in S_{2}}\left\|\operatorname{diag}\left(\alpha_{k}-\beta_{\sigma(k)}\right)\right\|$. That this is not a coincidence is the content of the next theorem.

Theorem 2. Let $A=A^{*}, B=B^{*} \in L(H)$. Let $\operatorname{sp} A=\left\{\alpha_{k}\right\}_{k=1}^{n}, \operatorname{sp} B=\left\{\beta_{k}\right\}_{k=1}^{n}$. Assume the numbering to satisfy $\left|\alpha_{1}\right| \geqslant \cdots \geqslant\left|\alpha_{n}\right|,\left|\beta_{1}\right| \leqslant \cdots<\left|\beta_{n}\right|$. Then,

$$
\|A+i B\| \geqslant\left\|\operatorname{diag}\left(\alpha_{k}+i \beta_{k}\right)\right\|=\max _{k}\left|\alpha_{k}+i \beta_{k}\right| \text {. }
$$

Proof. Let $\left\{e_{k}\right\}_{k=1}^{n}$ and $\left\{f_{k}\right\}_{k=1}^{n}$ be orthonormal bases of $H$ such that $A e_{k}=\alpha_{k} e_{k}$ and $B f_{k}=\beta_{k} f_{k}$ for all $k$. Let $M_{k}=\bigvee\left\{e_{l}: l \leqslant k\right\}$ and $N_{k}=\bigvee\left\{f_{l}: l \geqslant k\right\}$. Then, since $\operatorname{dim} M_{k}=k$ and $\operatorname{dim} N_{k}=n-k+1$, it follows that $\operatorname{dim}\left(M_{k} \cap N_{k}\right) \geqslant 1$. Let $x_{k}$ be a unit vector in $M_{k} \cap N_{k}$. The ordering of the $\alpha_{k}$ 's and the $\beta_{k}$ 's ensures that $\left\|A x_{k}\right\| \geqslant\left|\alpha_{k}\right|,\left\|B x_{k}\right\| \geqslant\left|\beta_{k}\right|$. Hence,

$$
\begin{aligned}
\left|\alpha_{k}+i \beta_{k}\right|^{2} & =\left|\alpha_{k}\right|^{2}+\left|\beta_{k}\right|^{2} \leqslant\left\|A x_{k}\right\|^{2}+\left\|B x_{k}\right\|^{2} \\
& =\frac{1}{2}\left[\left\|(A+i B) x_{k}\right\|^{2}+\left\|(A-i B) x_{k}\right\|^{2}\right] \\
& \leqslant \frac{1}{2}\left[\|A+i B\|^{2}+\|A-i B\|^{2}\right]=\|A+i B\|^{2} .
\end{aligned}
$$

(The first equality follows from the parallelogram identity, while the last equality is a consequence of the fact that $A-i B=(A+i B)^{*}$.) Hence,

$$
\|A+i B\| \geqslant \max _{k}\left|\alpha_{k}+i \beta_{k}\right| \text {. }
$$

The following is just a reformulation of Theorem 2: if $T \in L(H)$, then there exists a normal operator $N$ on $H$ such that (i) the real and imaginary parts of $N$ are, respectively, unitarily equivalent to the real and imaginary parts of $T$, and (ii) $\|N\| \leqslant\|T\|$.

\section{BIBLIOGRAPHY}

1. V. Lidskii, The proper values of the sum and product of symmetric matrices, Dokl. Akad. Nauk SSSR 75 (1950), 760-772.

2. H. Weyl, Das asymptotische Verteilungsgesetz der Eigenwerte linearer partielles Differentialgleichungen, Math. Ann. 71 (1912), 441-479.

3. H. Wielandt, An extremum property of sums of eigenvalues, Proc. Amer. Math. Soc. 6 (1955), 106-110.

School of Mathematics, Tata Institute of Fundamental Research, Homi Bhabha Road, BOMBAY 400 005, INDIA

Current address: Indian Statistical Institute, 7, S. J. S. Sansanwal Marg, New Delhi 110029, India 